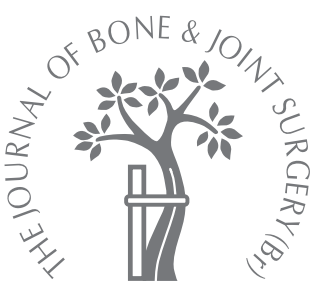

A. C. Day,

C. Kinmont, M. D. Bircher, S. Kumar

From St. George's Healthcare NHS

Trust, London, England

\title{
Crescent fracture-dislocation of the sacroiliac joint
}

\author{
A FUNCTIONAL CLASSIFICATION
}

Crescent fracture dislocations are a well-recognised subset of pelvic ring injuries which result from a lateral compression force. They are characterised by disruption of the sacroiliac joint and extend proximally as a fracture of the posterior iliac wing. We describe a classification with three distinct types. Type $I$ is characterised by a large crescent fragment and the dislocation comprises no more than one-third of the sacroiliac joint, which is typically inferior. Type II fractures are associated with an intermediate-size crescent fragment and the dislocation comprises between one- and two-thirds of the joint. Type III fractures are associated with a small crescent fragment where the dislocation comprises most, but not all of the joint. The principal goals of surgical intervention are the accurate and stable reduction of the sacroiliac joint. This classification proves useful in the selection of both the surgical approach and the reduction technique. A total of 16 patients were managed according to this classification and achieved good functional results approximately two years from the time of the index injury. Confounding factors compromise the summary short-form-36 and musculoskeletal functional assessment instrument scores, which is a well-recognised phenomenon when reporting the outcome of high-energy trauma.

Lateral compression fractures comprise up to $80 \%$ of all pelvic ring injuries. ${ }^{1-4}$ Of this group, crescent fractures form a less common subset, ${ }^{1-}$ 4 although the exact proportion is difficult to determine from the literature. The Pelvic and Acetabular Reconstruction Unit at St George's Healthcare NHS Trust provides a tertiary referral service. A review of our database identified 37 lateral compression-type pelvic ring injuries in a consecutive series of 118 fractures. A total of 14 may be classified as crescent fractures according to the definition of Borrelli et al. ${ }^{1,2}$ Therefore, crescent fractures are not uncommon and occur in approximately $12 \%$ of pelvic ring fractures admitted to a specialist unit. They are, in fact, fracture-dislocations of the sacroiliac joint in which there is variable disruption of the sacroiliac ligament complex, extending proximally as a fracture of the posterior iliac crest. A crescent-shaped segment of the iliac wing remains attached to the sacrum by the intact portion of the posterior ligament complex. ${ }^{1,4}$ The anteroposterior (AP) radiograph (Fig. 1) demonstrates the topography of a typical crescent fracture. The posterior pelvic ring injury is often accompanied by fractures of the pubic rami or a symphyseal diastasis. A three-dimensional (3D) reformat of spiral CT data of a crescent fracture is shown in Figure 2.

Crescent fractures are not commonly associated with life-threatening pelvic vascular injuries and the urgent application of a pelvic external fixator device in compression is not generally required. ${ }^{1-3,5,6}$ However, urogenital and vascular injuries can complicate any fracture or dislocation of the pelvic ring and should not be overlooked. The value of contrast-enhanced CT scans is established and facilitates the selection of patients who may benefit from angiography and arterial embolisation techniques. ${ }^{7}$

Operative intervention is recommended for the anatomical reduction and stable fixation of a crescent fracture-dislocation. Restoration of the normal anatomy should reduce the incidence of malunion, post-traumatic sacroiliac joint arthritis, painful stance phase gait cycle instability and seating obliquity. 5,6,8 Despite satisfactory reduction and stabilisation, some patients may experience persistent pain and dysfunction, which is commonly observed in high-energy injuries. ${ }^{9}$

A number of surgical techniques have been described. 1,2,5,9-16 To the authors' knowledge, there are only two published articles that spe- 


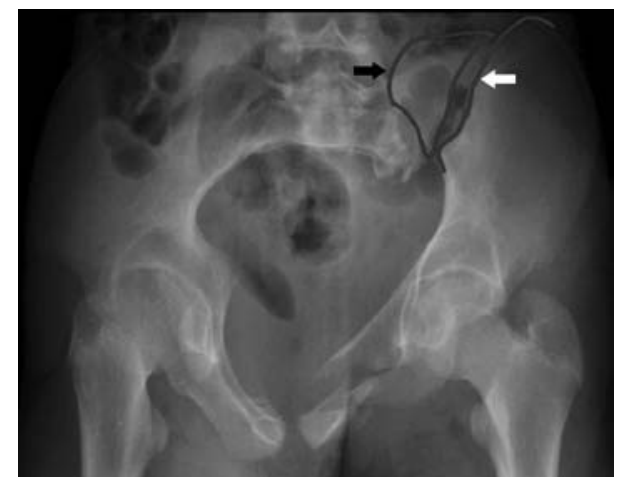

Fig. 1

Radiograph of a crescent fracture-dislocation of the left sacroiliac joint. The crescent fragment (black arrow) remains attached to the sacrum by the intact portion of the posterior sacroiliac ligament complex. The left hemipelvis (white arrow) is rotationally unstable, but vertical displacement is limited by the intact sacrospinous and sacrotuberous ligament complexes. $1,3,5$ There is an associated fracture of both the superior and inferior pubic ramus.

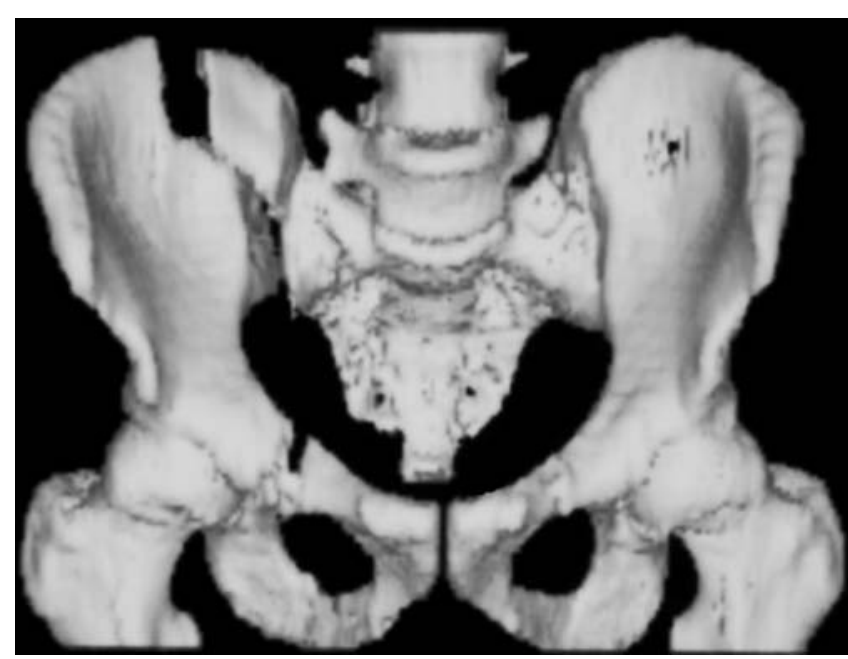

Fig. 2

This 3D reformat of spiral CT data demonstrates a crescent fracture of the right hemipelvis in which two-thirds of the sacroiliac joint was dislocated and is associated with a medium-sized crescent fragment.

cifically address the management of this fracture. Borrelli et $\mathrm{al}^{1,2}$ describe a fracture-dislocation involving approximately $50 \%$ of the sacroiliac joint, and restoration of a stable pelvic ring is advocated by means of a posterior subgluteal approach supplemented by a lag screw and plating technique. This approach is entirely appropriate for the type of fracture-dislocation described. ${ }^{1,2}$

There are a number of subtypes that may reasonably be described as crescent fractures. They share a common mechanism of injury and the fracture morphology is similar. Operative management is generally to be recommended, but, significantly, the operative approach and technique differ substantially for each subtype. We propose three distinct categories according to the extent of sacroiliac joint involvement, and the classification is inclusive of the fracture type described by Borrelli et al. ${ }^{1,2}$

Crescent fracture classification. Imaging should include plain radiographs comprising AP, inlet and outlet views. Additionally, spiral CT data sets should be reformatted in the axial plane with the scout grid lines orientated parallel to the superior end-plate of S1. Where appropriate software is available, $3 \mathrm{D}$ reformatting is particularly helpful in the radiological assessment of these injuries and should be orientated in projections similar to the plain radiograph series.

We identified three groups of crescent fracture according to the extent of sacroiliac joint involvement (Fig. 3).

Type I fractures involve less than one-third of the joint and enter it inferiorly. A large crescent fragment is observed and the fracture line enters the joint close to the foramen of the anterior S2 nerve root, a feature best appreciated from 3D CT data sets and the pelvic outlet view. This fracture type may be surgically addressed by means of an ilioinguinal approach. The lateral window usually gives sufficient exposure and facilitates an anterior plating technique, with direct visualisation of both the fracture and the sacroiliac joint.

Type II fractures involve between one-third and twothirds of the joint. The crescent fragment is of moderate size and the fracture line enters the joint between the anterior S1 and S2 foramina. This is, in effect, the fracture type described by Borrelli et al, ${ }^{1,2}$ and it may be surgically addressed by means of a posterior approach which facilitates the placement of interfragmentary screws and supplementary plates.

Borrelli et $\mathrm{al}^{1,2}$ comment that these lateral compression injuries may be associated with significant soft-tissue injuries, such as the Morel-Lavallee ${ }^{17}$ lesion.

Type III fractures involve more than two-thirds of the joint and are associated with a small, superior crescent fragment. The fracture line enters the joint posterior and superior to the anterior S1 nerve root foramen. This fracture type may therefore be surgically addressed by means of a closed or percutaneous reduction technique, supplemented by the placement of percutaneous iliosacral screws. The technique should only be undertaken by appropriately-trained practitioners, and in the case of delayed presentation, closed reduction may not be possible. Under these circumstances it may be argued that the lateral window of an ilioinguinal approach offers the best chance of accurate reduction with stable anterior plate fixation.

The principal fracture line is oblique, and its orientation will therefore vary according to the virtual gantry angle and the level of the axial CT cut. The S1 nerve root canal itself is oblique to the coronal and sagittal planes, and its appearance will therefore also vary according to similar criteria. The fracture type is determined not so much by the precise plane in which the fracture enters the sacroiliac joint at a carefully defined axial level, but rather by its overall morphology and the way in which this influences the choice of surgical approach. Examples of the three fracture types are shown in Figure 3, and are derived from axial reformats which demon- 


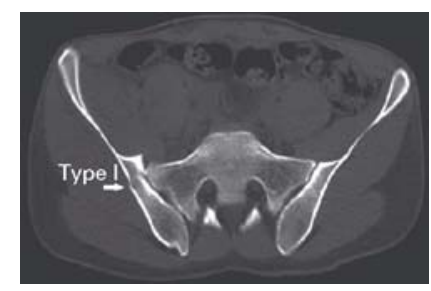

Fig. 3a

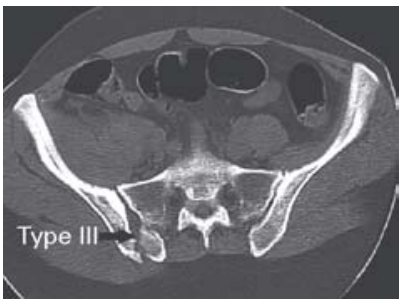

Fig. 3c

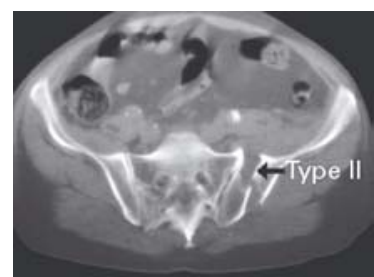

Fig. 3b

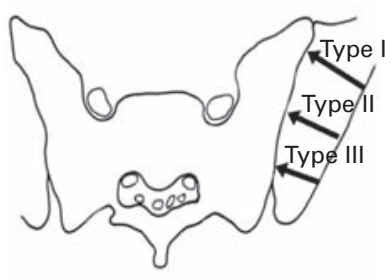

Fig. 3d
The positions of the principal fracture lines are shown for crescent fracture-dislocation types I, II and III, as defined by axial CT sections, reformatted parallel to the sacroiliac superior end-plate. a) Type I - the fracture enters the anterior third of the sacroiliac joint. b) Type II - the fracture enters the middle third of the sacroiliac joint. c) Type III- the fracture enters the posterior third of the sacroiliac joint. d) All three fracture types are shown diagrammatically.

strate the coronal plane in which the fracture enters the sacroiliac joint and its relationship to both the S1 nerve root canal and the sacral alar recess. It should be noted that the coronal plane in which the fracture enters the joint may differ from the fracture plane if the fracture is oblique to the joint, which is often the case.

\section{Patients and Methods}

Our classification system was applied to a consecutive series of 16 patients with crescent fractures admitted to St George's Healthcare NHS Trust between 1999 and 2001. One case was managed initially by non-operative means and presented late. The other cases were transferred within three weeks of injury. The mean age of the patient group was 25 years (16 to 63 ). There were eight males and eight females. Five patients sustained multiple musculoskeletal injuries, and of these, two sustained associated fractures of the acetabulum.

Pelvic external fixators had been applied in two cases at the referring hospital. The mechanism of injury was recorded as lateral compression in all cases, and there was no evidence of significant haemodynamic compromise. All of the patients were assessed at approximately two years after the index injury.

Radiological assessment. The fractures were evaluated by means of plain anteroposterior, inlet and outlet pelvic radiographs. For those cases associated with a fracture of the acetabulum, $45^{\circ}$ oblique views were obtained as described by Judet. ${ }^{18}$ The extent of each principal fracture line was assessed by spiral CT; $3 \mathrm{~mm}$ contiguous cuts were reformatted in an axial plane, parallel to the $\mathrm{S} 1$ superior end-plate, as determined from the lateral scout projection. Additional 3D reformatting was undertaken for selected cases in order to clarify the fracture morphology.

Post-operative assessment. Patients were reviewed both clinically and radiologically. Radiological criteria as assessed by plain AP, inlet and outlet projections, included the quality of fracture reduction and evidence of union.

CT scans were obtained if there was any doubt about fracture union. Clinical outcome was assessed by means of the validated short-form (SF) $36^{19}$ and musculoskeletal functional assessment instruments. ${ }^{20}$ These were administered between 12 and 18 months after surgery, by which time the patients were expected to be independently mobile, and fracture consolidation was anticipated. Ethical approval was obtained for the study. The data were rendered anonymous.

\section{Results}

Type I fractures. We identified four type I crescent fractures (Fig. 4). In addition to a crescent fracture, one patient sustained fractures of all four limbs, an ipsilateral fracture of the acetabulum involving both columns, a fracture of the ipsilateral pubic ramus and an extensive posterior MorelLavallee lesion. One patient sustained ipsilateral pubic ramus fractures, and the remaining two had a symphyseal diastasis.

Figure 4a illustrates a type I fracture. A large crescent fragment remains congruent with the sacroiliac joint and the superior portion of the ligament complex is intact. The axial CT scan (Fig. 4b) demonstrates a fracture of the iliac crest which enters the sacroiliac joint well within the anterior third, and the associated sacroiliac dislocation is noted. The inferior portion of the sacroiliac ligament complex may have been injured, but type I fractures cause the least ligamentous injury of the three subtypes. Traction was applied in flexion and the fracture was addressed through the lateral window of an ilioinguinal approach, controlling rotation with a Schanz pin, which was placed in the anteroinferior iliac spine by means of a percutaneous technique.

The united fracture and fixation in situ is illustrated in Figure 4c. The use of two plates is recommended, as stability in 6 degrees of freedom is required. In one case, the ilioinguinal approach was extended to address an acetabular fracture, and for another case a posterior approach was selected to avoid infected pin track sites following the removal of an external fixator device.

In the latter situation, the dissection required was more extensive than is typical of the classic crescent fracturedislocation described by Borrelli et al. ${ }^{1,2}$ It is for this reason that the lateral window of an ilioinguinal approach is preferred when the clinical circumstances allow. To summarise, three cases were managed through the lateral window of an ilioinguinal approach. This was extended in the first case to facilitate access to a fracture of the ipsilat- 


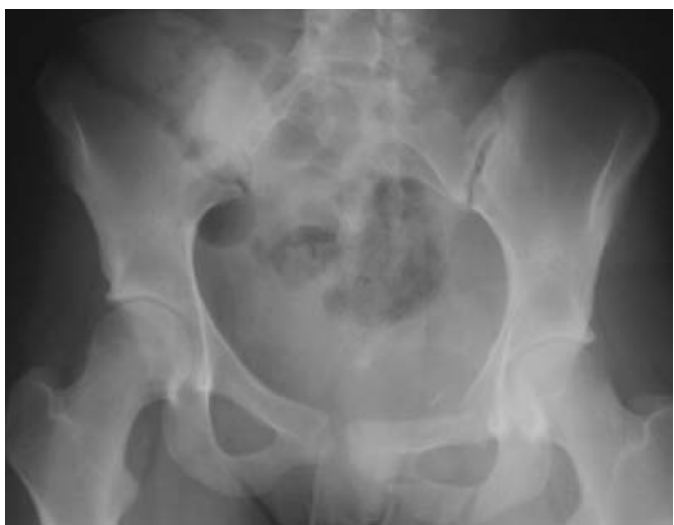

Fig. $4 \mathrm{a}$

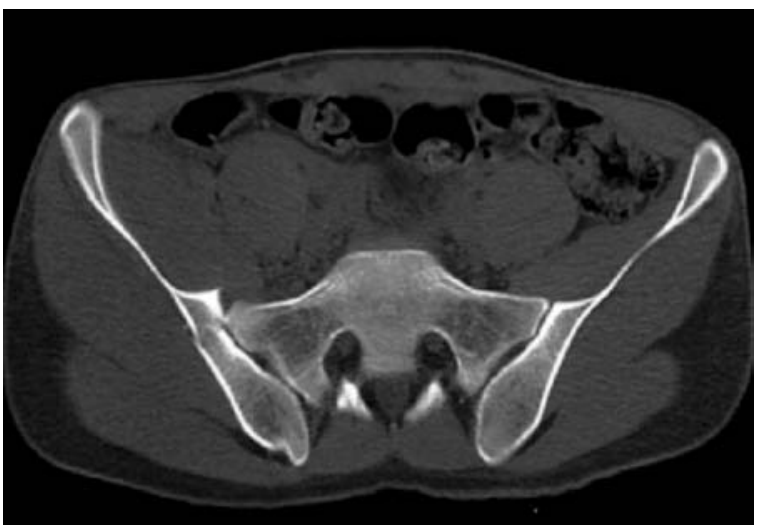

Fig. $4 b$

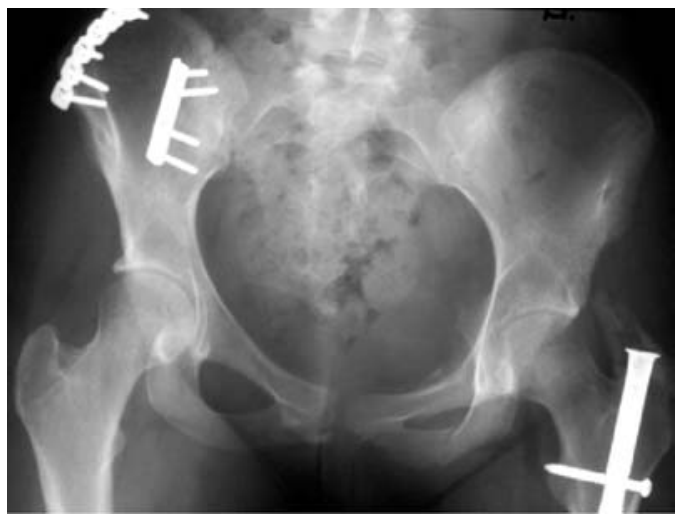

Fig. 4c

Type I crescent fracture. a) Anteroposterior radiograph, b) axial CT image and c) post-operative anteroposterior radiograph following union.

eral acetabulum, and an ipsilateral pubic ramus fracture was addressed simultaneously. An AO (Synthes, Stratec Medical, Oberdorf, Switzerland) external fixator device was applied in a second case, associated with fractures of all four pubic rami. The 'Delta' configuration was selected in distraction mode. This technique facilitates the reduction of bilateral pubic ramus fractures, sustained as a result of the internal rotation mechanism, and relies on the presence of an intact periosteal hinge. It provides reasonable stability without recourse to an extensive anterior approach. The third case was associated with a symphyseal diastasis which was stabilised by means of a four-hole AO (Synthes) large fragment reconstruction plate, using a separate Pfannenstiel incision. A posterior approach was selected for the remaining case to avoid infected external fixator pin sites. Sound union was achieved in all four cases.

Type II fractures. Of the four type II crescent fractures in this series, three were isolated pelvic fractures and all had pubic ramus fractures (Fig. 5). The fourth patient sustained multiple injuries and the crescent fracture was associated with a transverse fracture of the ipsilateral acetabulum. Figure 5a demonstrates a typical type II fracture. The axial CT reformat (Fig. 5b) demonstrates a fracture line which enters the middle third of the sacroiliac joint. Injury to the posterior sacroiliac ligament complex is more extensive in this case, and the crescent fragment is of intermediate size.

The fracture line, which is best appreciated on the axial CT scan, runs in a relatively oblique plane. A posterior approach was selected, as described by Borrelli et al. ${ }^{1,2}$ It was considered that an interfragmentary screw might result in shear rather than compression of the fracture, and the two ACE ribbon plates (DePuy Orthopaedics, Warsaw, Indiana) therefore incorporate interfragmentary screws, placed orthogonal to the fracture line (Fig. 5c). A further case (Fig. 5d) illustrates the classic technique, where it has been possible to place interfragmentary screws across an orthogonal fracture line with the addition of two contoured reconstruction plates (Synthes). Once again, the posterior approach was selected and proved satisfactory. In one case, the posterior fixation was supplemented by an external fixator device, applied in 'distraction mode' as described previously. If the pubic ramus fractures are well aligned, retrograde anterior column screws may be considered a less cumbersome alternative. It should be noted, however, that 


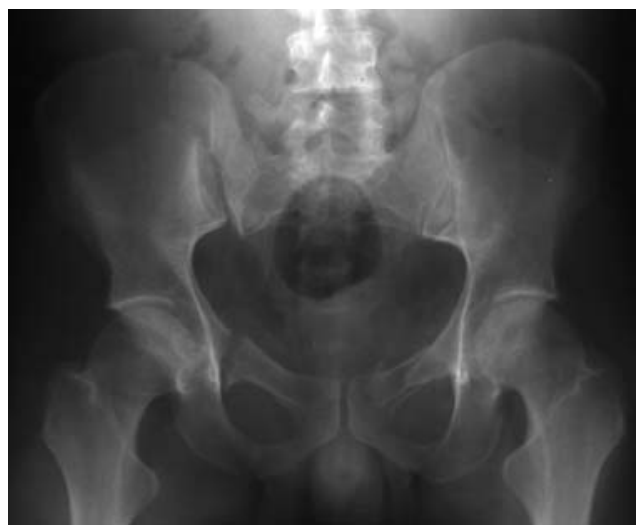

Fig. $5 a$

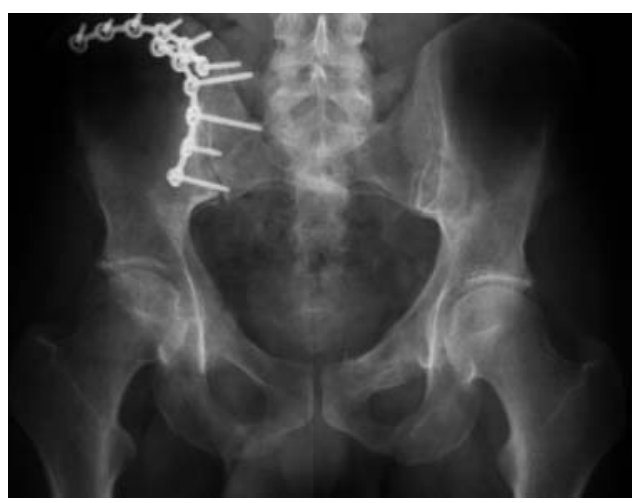

Fig. $5 c$

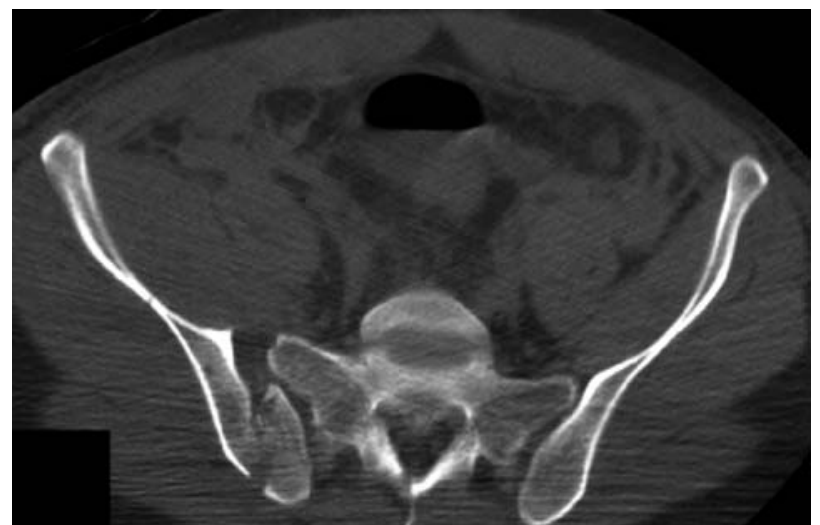

Fig. 5b

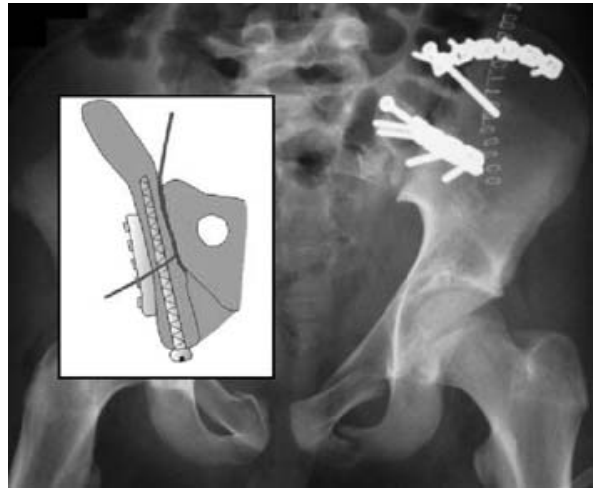

Fig. $5 d$ relli et al. ${ }^{1,2}$

Type II crescent fractures, a) anteroposterior radiograph, b) axial CT image, c) postoperative anteroposterior radiograph following union, and d) a further example demonstrating the classic technique described by Borrelli et al. ${ }^{1,2}$ The inset demonstrates the technique of internal fixation by means of an interfragmentary screw and neutralisation plate, as described by Bor-

satisfactory alignment cannot always be achieved. The patient sustaining an ipsilateral transverse fracture of the acetabulum required both ilioinguinal and KocherLangenbeck approaches. An anterior plating technique was therefore selected in respect of the crescent fracture.

In summary, three of the four cases were managed by means of the classic posterior approach and a technique similar to that described by Borrelli et al. ${ }^{1,2}$ All of the fractures in this group united uneventfully.

Type III fractures. Eight type III crescent fractures were identified. In one case there was an associated symphyseal diastasis, and pubic ramus fractures occurred in all of the other cases. A typical example is presented in Figure 6.

The now familiar pattern is observed in Figure 6, but in this case the dislocation encompasses more than two-thirds of the sacroiliac joint. The axial CT (Fig. 6b) highlights the small posterior crescent (white arrow) and the projected track of a percutaneous iliosacral screw (black arrow). These fractures are associated with more extensive disruption of the sacroiliac ligament complex, but are nevertheless distinct from pure sacroiliac dislocations and are consistently lateral compression injuries in which the degree of vertical displacement is limited. Six cases were treated with closed reduction and percutaneous iliosacral screw placement. This technique requires skeletal traction by means of a traction pin placed in the proximal tibia. Traction is applied with the hip in flexion on a specialised radiolucent pelvic reduction table (OSI, Union City, California) allowing correction of both the vertical and the posterior displacement. The internal rotation deformity may be corrected by means of a percutaneous Schanz pin, applied to either the anterior iliac crest or the anteroinferior iliac spine. The use of two Schanz pins facilitates fine adjustment of the reduction in 6 degrees of freedom, and translational reduction may be further supplemented by a ball spike pusher (Synthes, Stratec Medical).

The inlet, outlet and lateral projections required for this technique are then acquired by image intensifier, and navigation may prove to be a useful supplementary technique. The $3.2 \mathrm{~mm}$ guide wires for ACE (DePuy) $8 \mathrm{~mm}$ cannulated Timax screws, are then passed across the sacroiliac joint under image intensifier control. The use of a washer at the level of S1 is recommended. Stable reduction and internal fixation is therefore achieved by a minimally-invasive and indirect method. This technique requires specific training and regular surgical experience. Accurate closed reduction proved impossible in one case as a result of callus around the anterior column. The lateral two windows of an ilioinguinal approach were therefore selected.

An external fixator was applied as supplementary fixation in four cases. One further case was treated at the referring unit with an isolated external fixator. The case was then referred to 


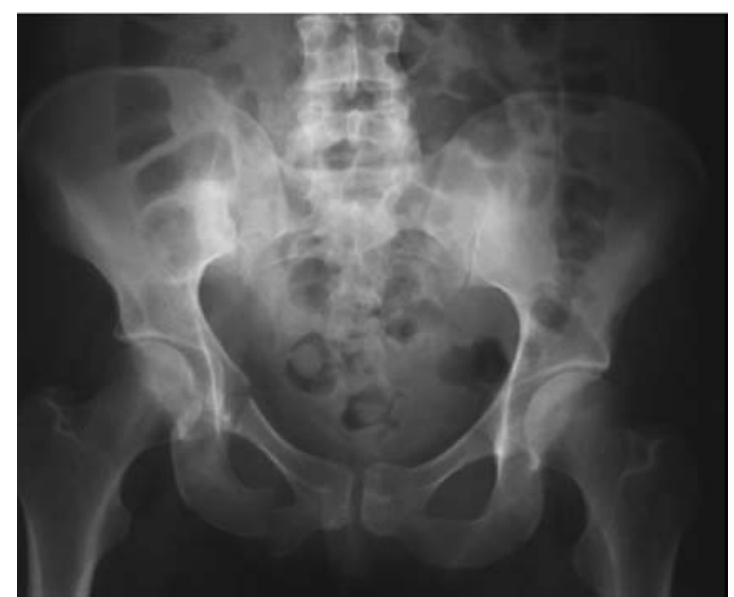

Fig. 6a

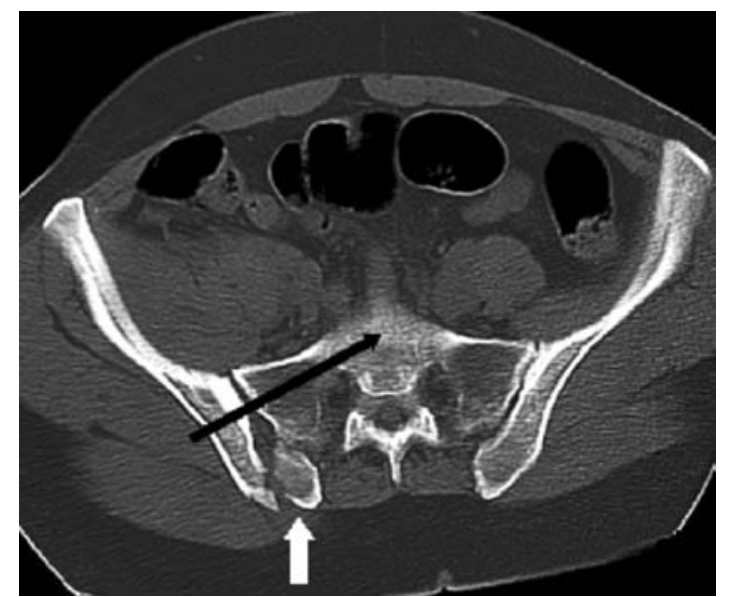

Fig. $6 b$

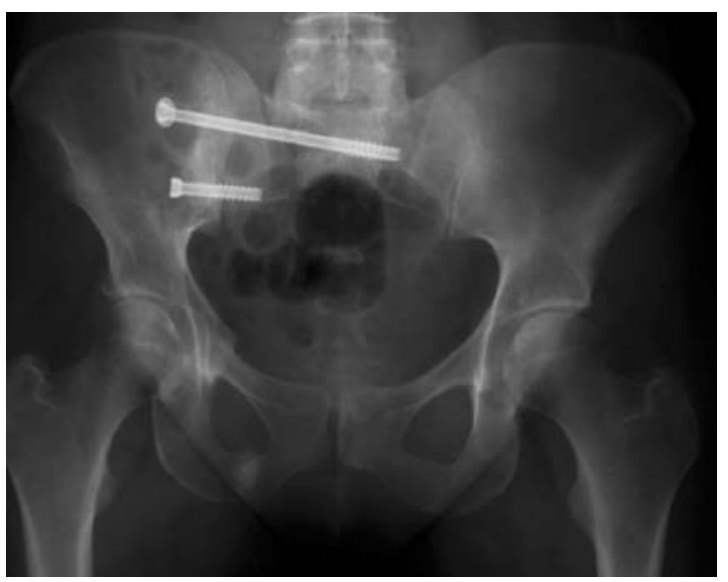

Fig. 6c

Type III crescent fracture. a) Anteroposterior radiograph, b) axial CT image showing a small crescent fragment (white arrow) and the track of an $\mathrm{S} 1$ level iliosacral screw (black arrow), and c) post-operative anteroposterior radiograph following union.

the specialist unit with pelvic obliquity four weeks after injury. Closed reduction proved impossible as a result of callus formation, and open reduction was precluded by the presence of pin track infections. A non-operative course of management was therefore selected and malunion occurred with approximately $2 \mathrm{~cm}$ of vertical displacement at the sacroiliac joint, and with a similar leg-length discrepancy. There was no significant pain, and as seating obliquity proved to be less of a problem, the patient elected to accept the result.

The SF-36 and musculoskeletal functional assessment instrument scores for the data set are presented in Figures 7 and 8 respectively.

\section{Discussion}

This series demonstrates the heterogeneous nature of crescent fractures. In all cases, lateral compression proves to be the mechanism of injury and rotational instability is observed. Some vertical displacement does occur, but it appears to be limited by the sacrospinous and sacrotuberous ligaments.
Operative stabilisation of crescent fractures is recommended in order to reduce the risk of malunion and pain. The suggested classification offers guidance on the choice of surgical approach. Type I fractures entering the anterior third of the sacroiliac joint may be addressed through the lateral window of an ilioinguinal approach. Type II fractures involving the middle third of the joint are generally addressed through a posterior approach. Type III fractures limited to the posterior third of the sacroiliac joint are often amenable to closed reduction and percutaneous iliosacral screw fixation.

Pelvic crescent fractures are a relatively uncommon subtype of lateral compression fracture and are rotationally unstable. There may be some limited vertical displacement, but in contrast to Tile type $\mathrm{C}$ fractures, the vertical displacement is limited by the sacrotuberous and sacrospinous ligaments, which typically remain intact. ${ }^{3,5}$ Operative intervention in these patients aims to achieve accurate reduction of the sacroiliac joint and stabilisation of the associated pelvic ring fractures or dislocations. This facilitates early 


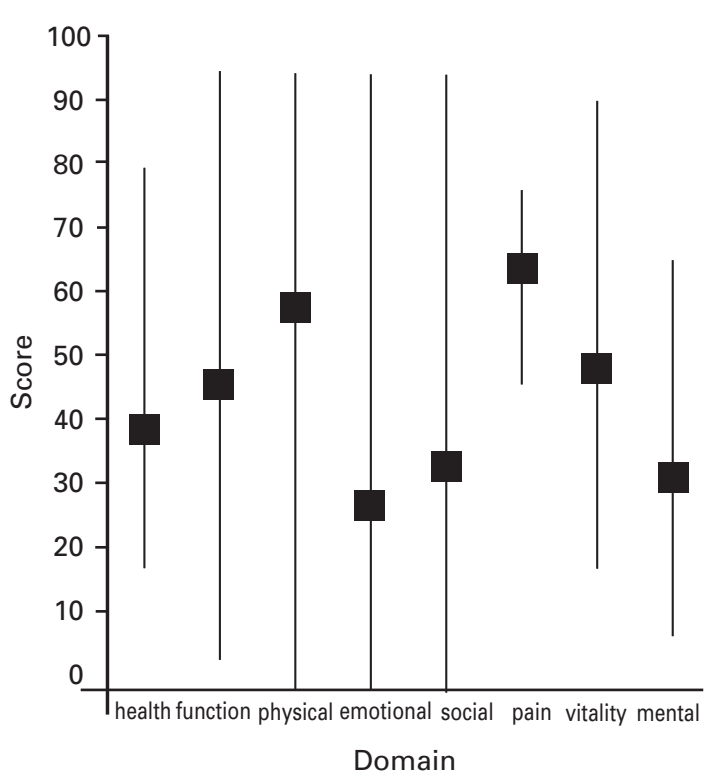

Fig. 7

Short-form-36 scores. The mean score and range are recorded for each domain. The lowest score represents the greatest level of disability.

mobilisation and minimises disability due to post-traumatic malunion and osteoarthritis or instability of the sacroiliac joint. ${ }^{1,2,5-7,10}$ We have observed rotational malalignment and limited vertical displacement, leading to leg-length discrepancy and seating obliquity in cases where nonoperative management proved necessary as a consequence of late referral. Burgess et $\mathrm{al}^{3}$ have observed horizontallyorientated fractures of the pubic rami in association with lateral compression injuries and vertically-orientated fractures in association with the AP compression and vertical shear injuries. Although the argument is intuitive, the orientation of pubic ramus fractures in this series proves heterogeneous and the recorded mechanism of injury is lateral compression in all cases. No significant case of pelvic vascular injury occurred in this series, which is consistent with the mechanism of injury, and similar findings have been reported previously. ${ }^{1-3,5,6}$ It should be emphasised, however, that bleeding may occur. ${ }^{17}$ It is therefore recommended that haemodynamic instability be treated on its merits. The application of an external fixator in compression mode should not be considered a reflex action, as contaminated pin sites may adversely affect the subsequent choice of surgical approach. An external fixator may, however, prove useful when applied in distraction mode, as described above. The technique takes advantage of the fact that the thick anterior periosteum around the pubic rami remains largely intact and may serve as a tension band.

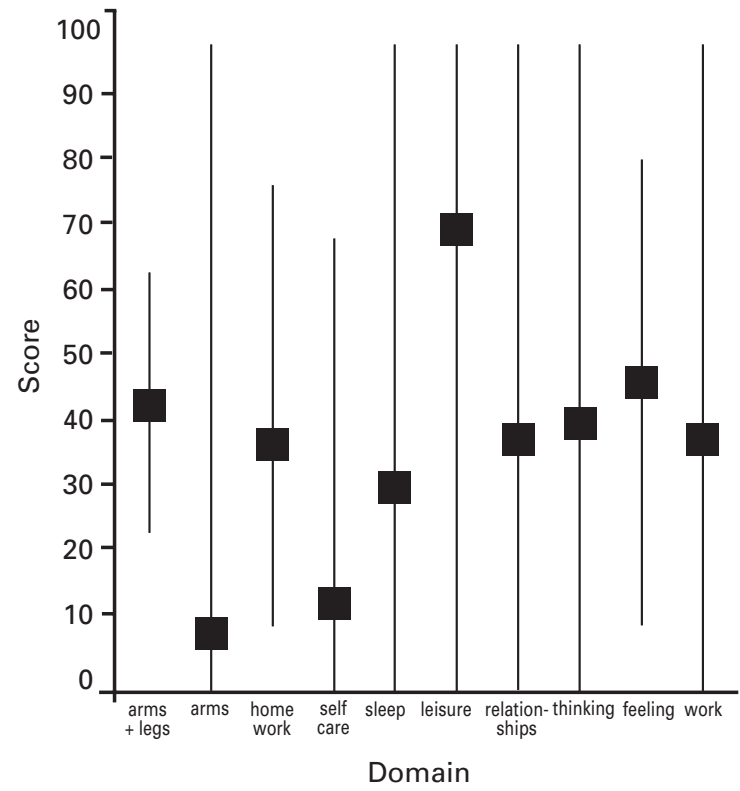

Fig. 8

Musculoskeletal functional assessment instrument scores. The mean score and range are recorded for each domain. The highest score represents the greatest level of disability.

In the absence of haemodynamic instability, it makes sense to apply this device at the same time as definitive surgical management.

A number of surgical techniques have been described for the reduction and stabilisation of sacroiliac fracturedislocations. Each has merit, but the variety of techniques suggests a variety of fracture patterns. Only two published articles specifically address the morphology and surgical management of crescent fractures. ${ }^{1,2}$ The posterior approach as described by Borrelli et $\mathrm{al}^{1,2}$ is considered to be a safe and reliable method of fixation in type II fractures. The crescent fragment will be of sufficient size to allow the insertion of stable inter-table lag screws and supplementary plates without the need for excessive soft-tissue stripping. On the other hand, type III fractures are associated with small crescent fragments and are less amenable to stable fixation by this technique. Supplementary transarticular fixation may be required, ${ }^{1,2,15}$ and closed degloving injuries such as the Morel-Lavallee lesion ${ }^{17}$ may be present, thereby increasing the surgical risk. We suggest that these injuries may be better managed by percutaneous iliosacral screw fixation. Although technically demanding, percutaneous iliosacral screws require minimum soft-tissue dissection and allow stable reduction of the sacroiliac dislocation in the presence of very small crescent fragments. ${ }^{14,16}$ The iliac vessels, together with the L5, S1 and S2 nerve roots, are theoretically at risk. This method may be usefully supple- 
mented by surgical navigation techniques. Proponents of the anterior approach for sacroiliac joint fixation stress the importance of visualising the anterior face of the joint as an aid to accurate reduction. ${ }^{11,14}$ The posterior approach does not allow the surgeon to assess joint congruence accurately, and relies on an indirect reduction technique which may be compromised by plastic deformation, comminution, and small key-in areas.

The anterior approach (effectively the lateral window of an ilioinguinal approach) is well suited to type I fractures. The L5 nerve root running in the lumbosacral trunk must be respected. ${ }^{1,2,4}$

This classification is pragmatic in that it facilitates the selection of an appropriate approach and surgical technique for each fracture within a heterogeneous group. Surgeons, should, however, remain flexible, as the choice of approach may also be influenced by associated soft-tissue injuries, contaminated external fixator pin sites, an ipsilateral acetabular fracture, or a delay in referral. All fractures in this series achieved union, and malunion was prevented in the cases which were managed operatively. Despite satisfactory technical outcome measures, the functional assessment scores demonstrate significant residual disability throughout the groups and within domains. There is no evidence that the three fracture types have different functional outcomes, and there is no obvious relationship between age and outcome, although the maximum age in the series was only 63. All of the fractures united with only one malunion. This case was managed non-operatively for reasons described in the text. All patients returned to work, but five with multiple injuries including two with ipsilateral acetabular fractures and the one with malunion, recorded a higher level of residual disability on SF-36 and musculoskeletal functional assessment instrument scores, particularly for the role physical and pain domains in the case of the SF-36. The leisure and recreational activity domains were the worst affected for the musculoskeletal functional assessment instrument.

Overall, the highest mean levels of dysfunction occurred in the role physical and pain domains for the SF-36 and the leisure and recreational activities for the musculoskeletal functional assessment instrument. Although the technical outcome following surgery was satisfactory in other published series, pain requiring regular analgesia and a return to less strenuous employment are commonly reported. ${ }^{5,10}$ The use of functional assessment instruments is clearly beneficial in determining the outcome, in view of the fact that the majority of these patients achieve good or excellent results on the basis of radiographs and clinical assessment alone. This phenomenon is now well accepted in the assessment of trauma, and in particular where there are multiple injuries.

Scores demonstrating the highest overall level of dysfunction in this series relate to those patients with failure of fixa- tion, multiple injuries and malunion following non-operative management.

The outcome may therefore be optimised by accurate reduction and stable internal fixation by means of an appropriate approach. The objective of surgical management is to prevent malunion which may result in pelvic obliquity and pain. Surgeons may find this classification helpful in the selection of an appropriate surgical technique.

No benefits in any form have been received or will be received from a commercial party related directly or indirectly to the subject of this article.

\section{References}

1. Borrelli J, Koval KJ, Helfet DL. The crescent fracture: a posterior fracture dislocation of the sacroiliac joint. J Orthop Trauma 1996;10:165-70.

2. Borrelli J Jr, Koval KJ, Helfet DL. Operative stabilization of fracture dislocations of the sacroiliac joint. Clin Orthop 1996;329:141-6.

3. Burgess AR, Eastridge BJ, Young JW, et al. Pelvic ring disruptions: effective classification system and treatment protocols. J Trauma 1990;30:848-56.

4. Dalal SA, Burgess AR, Young JW, et al. Pelvic fracture in multiple trauma: classification by mechanism is key to pattern of organ injury, resuscitative requirements and outcome. J Trauma 1989;29:981-1000.

5. Tile M. Pelvic fractures: operative versus non-operative treatment. Orthop Clin North Am 1980;11:423-64.

6. Holdsworth FW. Dislocation and fracture-dislocation of the pelvis. J Bone Joint Surg [Br] 1948;30-B:461-6.

7. Stephen DJ, Kreder HJ, Day AC, et al. Early detection of arterial bleeding in acute pelvic trauma. J Trauma 1999;47:638-42.

8. Dujardin FH, Hossenbaccus M, Duparc F, Biga N, Thomine JM. Long-term functional prognosis of posterior injuries in high-energy pelvic disruption. $J$ Orthop Trauma 1998:12:145-50.

9. Pattee GA, Bohlman HH, McAfee PC. Compression of a sacral nerve as a complication of screw fixation of the sacro-iliac joint: a case report. J Bone Joint Surg [Am] 1986;68-A:769-71.

10. Kellam JF, McMurtry RY, Paley D, Tile M. The unstable pelvic fracture: operative treatment. Orthop Clin North Am 1987;18:25-41.

11. Simpson LA, Waddell JP, Leighton RK, Celom JG, Tile M. Anterior approach and stabilization of the disrupted sacroiliac joint. J Trauma 1987;27:1332-9.

12. Mears DC, Capito CP, Deleeuw A. Post-pelvic disruptions managed by the use of the cobra plate. Instr Course Lect 1988;37:143-50.

13. Dabezies SA, Millet CW, Murphy CW, et al. Stabilisation of sacroiliac joint disruption with threaded compression rods. Clin Orthop 1989;246:165-71.

14. Lange RH, Webb LX, Mayo KA. Efficacy of the anterior approach for fixation of sacroiliac dislocations and fracture dislocations [abstract]. J Orthop Trauma 1990;4:220-21

15. Routt MLC Jr, Simonia PT, Mills WJ. Iliosacral screw fixation: early complications of the percutaneous technique. J Orthop Trauma 1997;11:584-9.

16. Starr AJ, Walter JC, Harris RW, Reinert CM, Jones AL. Percutaneous screw fixation of fractures of the iliac wing and fracture-dislocations of the sacro-iliac joint (OTA Types 61-B2.2 and 61-B2.3, or Young-Burgess "lateral compression Type 11" pelvic fractures). J Orthop Trauma 2002;16:116-23.

17. Tseng S, Tornetta P. Percutaneous management of Morel-Lavallee lesions. J Bone Joint Surg [Am] 2006;88-A:92-105.

18. Judet R. Radiology of the normal acetabulum. In: Letournel E, Judet R eds. Fractures of the acetabulum. Second ed. Berlin: Springer Verlag, 1993.

19. Garratt AM, Ruta DA, Abdalla MI, Buckingham JK, Russell IT. The SF36 health survey questionnaire: an outcome measure suitable for routine use within the NHS? BMJ 1993;306:1440-4.

20. Engelberg R, Martin DP, Agel J, et al. Musculoskeletal function assessment instrument: criterion and construct validity. J Orthop Res 1996;14:182-92. 